by the $\mathrm{C}$ atom $[\mathrm{C}(5)]$ that is bound to the ester group. The orientation of the ester group with respect to the benzene ring would be expected to be anti- or synperiplanar because only these two conformations would allow resonance of the carbonyl group with the benzene $\pi$ system. It is found that the ester group is out of plane by $103(1)^{\circ}$ and thus is not stabilized by resonance. This is confirmed by the bond lengths of the carbonyl $\mathrm{C}$ to $\mathrm{O}[\mathrm{C}(8)-\mathrm{O}(2)]$ and to the ring $\mathrm{C}[\mathrm{C}(8)-\mathrm{C}(5)]$, which are $1 \cdot 19(1)$ and 1.50 (1) $\AA$, respectively. Both bond lengths are typical for a $\mathrm{C}-\mathrm{O}$ double bond and a $\mathrm{C}-\mathrm{C}$ single bond. The out-of-plane orientation of the ester group with respect to the benzene ring must be due to steric hindrance by the two bulky groups, the pyrrolidine ring and the $\mathrm{Cl}$ atom, which are ortho to the ester group.

A relatively short bond length of a $1.34 \AA$ is shown by the $\mathrm{C}(8)-\mathrm{O}(1)$ single bond. In 3-hydroxy-5phenylisoxazole the $\mathrm{C}-\mathrm{O}$ single bond showed the same distance (Marongiu, Biagini \& Carmas, 1969). $C(9)$ and $C(10)$ have unusually large thermal parameters, and slight positional disorder may explain the unusually short $\mathrm{C}(9)-\mathrm{C}(10)$ distance of 1.447 (8) $\AA$.

The Nicolet $R 3 / m$ X-ray diffractometer and crystallographic computing system at Washington State
University were purchased with funds provided by the National Science Foundation (Grant CHE-8408407) and the Boeing Corporation. The authors thank Dr Roger Willett of Washington State University for technical assistance and the M. J. Murdock Charitable Trust of Research Corporation and the National Institutes of Health (Biomedical Research Support Grant No. 2-S07-RR07170-06) for financial support.

\section{References}

Campana, C. F., Shepard, D. F. \& Litchman, W. M. (1980). Inorg. Chem. 20, 4039-4043.

Harris, R. L. N., Huppatz, J. L. \& Phillips, J. N. (1975). J. Aust. Chem. 30, 2213-2217.

International Tables for X-ray Crystallography (1974). Vol. IV, pp. 55, 99. Birmingham: Kynoch Press. (Present distributor Kluwer Academic Publishers, Dordrecht.)

MCMurRY, J. E. (1973). Org. Synth. 53, 59-62.

Marongiu, G., Biagini, S. \& Carmas, M. (1969). Acta Cryst. B25, 2108-2112.

Natale, N. R. (1982). Tetrahedron Lett. pp. 5009-5012.

Natale, N. R., McKenna, J. I., Niou, C.-S., BoRTH, M. L. \& HOPE, H. (1985). J. Org. Chem. 50, 5660-5666.

QunCY, D. A. \& NATALE, N. R. (1985). Unpublished observations.

Schlessinger, R. H., Doss, M. A. \& Richardson, S. (1986). J. Am. Chem. Soc. 108, 3112-3113.

SHELDRICK, G. M. (1984). SHELXTL Users Manual, revision 4.1. Nicolet XRD Corporation, Madison, Wisconsin, USA.

Acta Cryst. (1989). C45, 1563-1565

\title{
X-ray Structure of a (D-galacto-Pentaacetoxypentyl)pyrazoline
}

\author{
By C. F. Conde AND A. Conde \\ Departamento de Fisica de la Materia Condensada, Instituto de Ciencias de Materiales, \\ Universidad de Sevilla-CSIC. Apartado 1065, 41080 Sevilla, Spain
}

(Received 9 December 1988; accepted 9 February 1989)

\begin{abstract}
Dimethyl-3-nitro-1-pyrazolin-4-yl)penta- $O$-acetyl-D-galacto-pentitol, $\mathrm{C}_{20} \mathrm{H}_{29} \mathrm{~N}_{3} \mathrm{O}_{12}, M_{r}$ $=503 \cdot 5$, orthorhombic, $P 22_{1} 2_{1} 2_{1}, a=14.471$ (9), $b=$ 14.518 (3), $c=12.028(2) \AA, V=2527.0(1 \cdot 7) \AA^{3}, Z$ $=4, D_{x}=1.323 \mathrm{Mg} \mathrm{m}^{-3}, \lambda(\mathrm{Mo} K \alpha)=0.7107 \AA, \mu$ $=0.10 \mathrm{~mm}^{-1}, F(000)=1064$, room temperature, final $w R=0.035$ for 1836 observed reflexions. Bond distances and angles are all within the expected ranges. The pyrazoline ring exhibits approximate mirror symmetry and a puckering amplitude of 0.244 (6) $\AA$. Torsion angles of the acetoxy groups with the sugar chain range from 33 to $72^{\circ}$. Crystal cohesion is mainly due to van der Waals interactions but there are two intermolecular hydrogen bonds
\end{abstract}

0108-2701/89/101563-03\$03.00 linking molecules related by screw axes along [100] and [001].

Introduction. The structure determination of 1-(3,5dimethyl-3-nitro-1-pyrazolin-4-yl)-penta- $O$-acetyl-Dgalacto-pentitol was undertaken as part of a continuing research project on $\mathrm{C}$-nucleosides and precursors. The title compound was prepared as an intermediate product in the synthesis of (pentaacetoxypentyl)pyrazoles (III) with the sugar nitroolefins (I) as dipolarophiles. Initially, the cycloadditions gave the pyrazolines (II), which were aromatized to the pyrazoles (Mancera, Rodriguez, Roffe \& Galbis, 1988). The stereochemistry was (C) 1989 International Union of Crystallography 
tentatively assigned on the basis of chemical and spectroscopic data and an X-ray study of the title compound was suggested to confirm the proposed configuration.

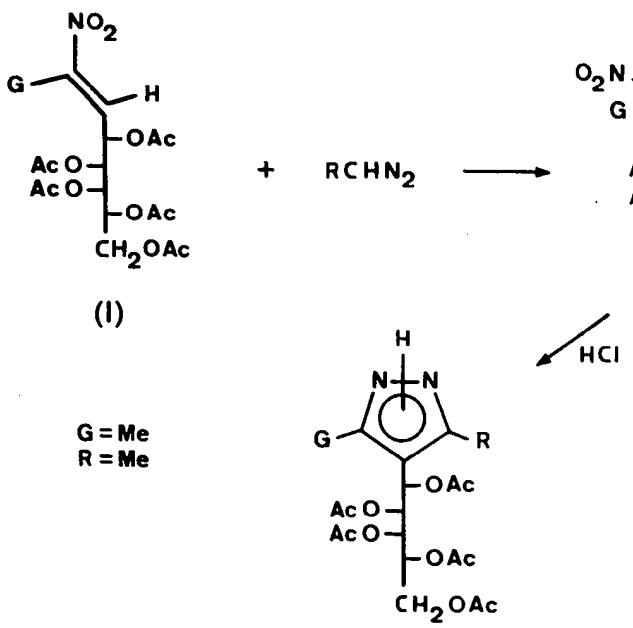

(III)

Experimental. Single crystals in the form of colourless needles elongated along [001] prepared in the Organic and Pharmaceutical Chemistry Department of the University of Sevilla. Cell parameters were refined by least-squares calculations from 25 reflexions with $5<\theta<20^{\circ}$ on an Enraf-Nonius CAD-4 diffractometer. Intensity data from a crystal of dimensions $0.20 \times 0.15 \times 0.30 \mathrm{~mm}$ were collected up to $\sin \theta / \lambda=0.60 \AA^{-1}$ with an $\omega-2 \theta$ scan using graphite-monochromated Mo $K \alpha$ radiation. Two reflexions $(41 \overline{3}, 34 \overline{3})$ monitored periodically during data collection showed that the crystal was stable to $\mathrm{X}$-rays. Index ranges of unique data were $0 \leq h \leq 17$, $0 \leq k \leq 17,0 \leq l \leq 14$. 2476 reflexions were collected of which 1836 unique reflexions were considered observed $[I>2 \sigma(I)]$. The structure was solved by direct methods using MULTAN80 (Main et al., 1980). All the $\mathbf{H}$ atoms were located from a difference Fourier map. Full-matrix least-squares refinement with non- $\mathrm{H}$ atoms refined anisotropically and $\mathrm{H}$ atoms isotropically converged at $R=0.048$ and $w R=0.035, S$ (goodness of fit) $=1.38$. The function minimized was $\sum w\left(\left|F_{o}\right|-\left|F_{c}\right|\right)^{2}$ where $w=$ $1 / \sigma^{2}(F)$. Maximum shift/e.s.d. ratio for non-H atoms $=0.02$. Residual electron densities in the final difference Fourier map were within 0.20 and -0.25 e $\AA^{-3}$. The atomic scattering factors were from International Tables for X-ray Crystallography (1974). Crystallographic programs of the XRAY76 system (Stewart, Machin, Dickinson, Ammon, Heck \& Flack, 1976) were used throughout.

Discussion. The final positional and equivalent isotropic thermal parameters for the non-H atoms are
Table 1. Atomic coordinates and thermal parameters $\left(\AA^{2} \times 10^{3}\right)$

$$
U_{\mathrm{eq}}=\frac{1}{3} \sum_{i} \sum_{j} U_{i j} a_{i}^{*} a_{j}^{*} \mathbf{a}_{i} \cdot \mathbf{a}_{j} \cdot \cos \left(\mathbf{a}_{i}, \mathbf{a}_{j}\right) .
$$

\begin{tabular}{|c|c|c|c|c|}
\hline & $x$ & $y$ & $z$ & $U_{\mathrm{eq}}$ \\
\hline 011 & 0.7034 (4) & $0.0799(5)$ & 0.4340 (4) & $110(3)$ \\
\hline 012 & $0.5870(3)$ & $0 \cdot 1282(4)$ & $0.3367(6)$ & $125(3)$ \\
\hline 041 & 0.9479 (2) & 0.1552 (2) & $0 \cdot 1666(3)$ & 38 (1) \\
\hline 042 & 0.9541 (3) & $0.0878(3)$ & $-0.0011(4)$ & $82(3)$ \\
\hline 051 & 0.8581 (2) & $0.2748(2)$ & $0 \cdot 3043$ (3) & 39 (1) \\
\hline O52 & $0.7195(3)$ & 0.2937 (3) & 0.3777 (3) & $74(2)$ \\
\hline 061 & $0.8288(2)$ & $0.2989(2)$ & $0.0060(3)$ & 37 (1) \\
\hline O62 & $0.9735(3)$ & $0.3119(4)$ & $-0.0542(4)$ & 83 (2) \\
\hline O71 & $0.7276(2)$ & $0.4241(2)$ & $0 \cdot 1464(3)$ & $39(1)$ \\
\hline O72 & $0.7322(3)$ & $0.5085(3)$ & $0.3032(3)$ & $73(2)$ \\
\hline 081 & 0.8549 (3) & $0.5805(3)$ & $0.0970(3)$ & 47 (2) \\
\hline O82 & 0.9849 (3) & $0.6179(3)$ & $0.0056(4)$ & $82(2)$ \\
\hline N1 & $0.7776(4)$ & $-0.0815(3)$ & $0.2382(5)$ & $66(2)$ \\
\hline N2 & $0.7030(4)$ & $-0.0435(3)$ & $0.2555(5)$ & $64(2)$ \\
\hline N11 & $0.6620(4)$ & 0.0931 (4) & $0.3463(6)$ & $73(3)$ \\
\hline $\mathrm{Cl}$ & 0.7095 (4) & 0.0593 (4) & $0.2396(5)$ & $47(2)$ \\
\hline $\mathrm{C} 2$ & 0.8148 (4) & $0.0800(4)$ & $0.2445(5)$ & $36(2)$ \\
\hline C3 & 0.8531 (4) & $-0.0162(4)$ & $0.2128(5)$ & $44(2)$ \\
\hline C4 & $0.8475(4)$ & $0 \cdot 1554(4)$ & $0 \cdot 1645(5)$ & $34(2)$ \\
\hline C5 & 0.8187 (4) & $0.2526(4)$ & $0.1966(5)$ & $29(2)$ \\
\hline C6 & $0.8580(4)$ & $0.3246(4)$ & $0 \cdot 1175(5)$ & $30(2)$ \\
\hline C7 & 0.8269 (4) & 0.4219 (4) & $0 \cdot 1416(5)$ & $33(2)$ \\
\hline C8 & $0.8622(5)$ & $0.4879(4)$ & 0.0534 (5) & $46(2)$ \\
\hline C11 & $0.6584(5)$ & $0.0830(6)$ & $0 \cdot 1366(9)$ & 89 (4) \\
\hline C31 & $0.9366(6)$ & $-0.0456(5)$ & $0.2806(9)$ & $83(4)$ \\
\hline C41 & $0.9916(4)$ & $0.1219(5)$ & $0.0751(6)$ & $55(3)$ \\
\hline $\mathrm{C} 42$ & $1.0941(5)$ & $0 \cdot 1365(7)$ & $0.0878(7)$ & $76(4)$ \\
\hline C51 & $0.8013(5)$ & 0.2944 (5) & $0 \cdot 3887(5)$ & $48(3)$ \\
\hline C52 & $0.8500(6)$ & $0.3177(7)$ & $0.4900(7)$ & $88(4)$ \\
\hline C61 & 0.8955 (5) & $0.2946(4)$ & $-0.0732(5)$ & $51(3)$ \\
\hline $\mathrm{C} 62$ & $0.8558(6)$ & $0.2671(6)$ & $-0.1820(6)$ & $72(3)$ \\
\hline C71 & $0.6886(4)$ & 0.4695 (4) & $0.2347(5)$ & $45(2)$ \\
\hline $\mathrm{C} 72$ & $0.5857(5)$ & $0.4601(6)$ & $0.2330(7)$ & $73(3)$ \\
\hline $\mathrm{C} 81$ & $0.9215(5)$ & 0.6404 (4) & $0.0632(6)$ & $52(3)$ \\
\hline C82 & $0.9036(6)$ & $0.7351(5)$ & $0.1006(6)$ & $65(3)$ \\
\hline
\end{tabular}

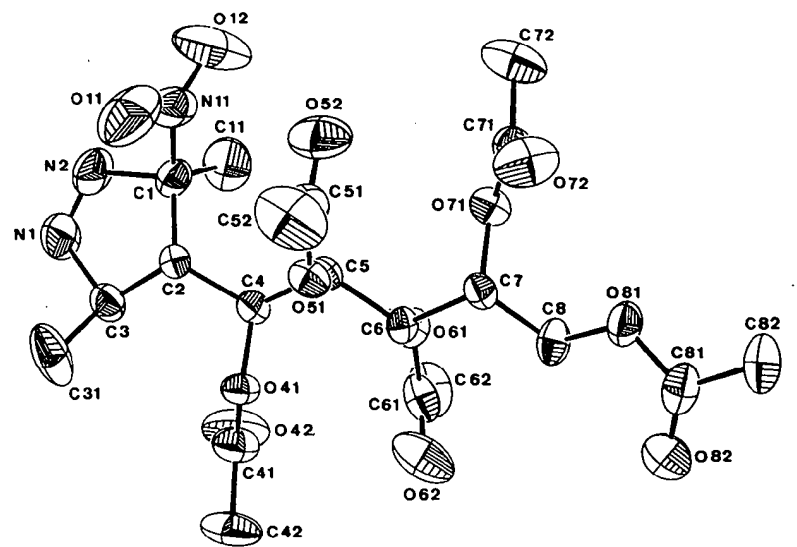

Fig. 1. An $O R T E P$ view of the molecule with atom labelling.

given in Table 1.* An ORTEP (Johnson, 1965) plot of the molecule with atomic labelling is displayed in Fig. 1. Bond lengths and angles involving non-H atoms are listed in Table 2 and the values are all within the expected ranges.

The puckering of the pyrazoline ring can be described (Cremer \& Pople, 1975) by a puckering

* Lists of structure factors, anisotropic thermal parameters and H-atom parameters have been deposited with the British Library Document Supply Centre as Supplementary Publication No. SUP 51944 (13 pp.). Copies may be obtained through The Executive Secretary, International Union of Crystallography, 5 Abbey Square, Chester $\mathrm{CH} 12 \mathrm{HU}$, England. 
Table 2. Bond distances $(\AA)$ and angles $\left({ }^{\circ}\right)$ involving non- $\mathrm{H}$ atoms

\begin{tabular}{|c|c|c|c|}
\hline $062-\mathrm{C} 61$ & $1.179(9)$ & $\mathrm{C} 62-\mathrm{C} 61$ & $1.484(10)$ \\
\hline $\mathrm{C} 61-061$ & $1.358(8)$ & $\mathrm{O} 82-\mathrm{C} 81$ & $1 \cdot 195(9)$ \\
\hline $\mathrm{C} 31-\mathrm{C} 3$ & 1.519 (11) & $\mathrm{C} 82-\mathrm{C} 81$ & $1.470(10)$ \\
\hline C4-O41 & $1.453(6)$ & $\mathrm{C} 4-\mathrm{C} 5$ & $1.521(8)$ \\
\hline $\mathrm{C} 4-\mathrm{C} 2$ & $1.532(8)$ & $\mathrm{O} 51-\mathrm{C} 51$ & $1.337(7)$ \\
\hline $\mathrm{O} 51-\mathrm{C} 5$ & $1.452(6)$ & O71-C7 & $1.440(6)$ \\
\hline O71-C71 & $1.371(7)$ & $\mathrm{C} 6-\mathrm{O} 61$ & $1.454(7)$ \\
\hline $\mathrm{C} 6-\mathrm{C} 7$ & $1.511(8)$ & $\mathrm{C} 6-\mathrm{C} 5$ & $1.524(8)$ \\
\hline $\mathrm{C} 7-\mathrm{C} 8$ & $1.517(8)$ & $\mathrm{O} 41-\mathrm{C} 41$ & $1.359(8)$ \\
\hline $\mathrm{C} 51-052$ & $1 \cdot 191(8)$ & $\mathrm{C} 51-\mathrm{C} 52$ & 1.448 (11) \\
\hline $\mathrm{C} 8-081$ & $1.447(7)$ & $\mathrm{O} 81-\mathrm{C} 81$ & $1.361(8)$ \\
\hline $\mathrm{C}^{3}-\mathrm{C} 2$ & $1.550(8)$ & $\mathrm{C} 3-\mathrm{N} 1$ & $1.478(8)$ \\
\hline $\mathrm{C} 42-\mathrm{C} 41$ & $1.507(10)$ & $\mathrm{C} 2-\mathrm{Cl}$ & $1.553(8)$ \\
\hline $\mathrm{C} 41-\mathrm{O} 42$ & $1.174(8)$ & $\mathrm{N} 1-\mathrm{N} 2$ & $1.230(7)$ \\
\hline $\mathrm{O} 72-\mathrm{C} 71$ & $1 \cdot 182(7)$ & $\mathrm{C} 72-\mathrm{C} 71$ & $1.495(9)$ \\
\hline $\mathrm{Cl}-\mathrm{N} 2$ & $1.507(7)$ & $\mathrm{Cl}-\mathrm{N} 11$ & $1.536(10)$ \\
\hline $\mathrm{Cl}-\mathrm{Cll}$ & 1.484 (12) & N11-OI1 & $1.229(9)$ \\
\hline $\mathrm{N} 11-\mathrm{O} 12$ & $1 \cdot 204(8)$ & & \\
\hline $\mathrm{O} 62-\mathrm{C} 61-\mathrm{C} 62$ & $126 \cdot 8(7)$ & $\mathrm{C} 62-\mathrm{C} 61-\mathrm{O} 61$ & $110.8(6)$ \\
\hline $\mathrm{O} 62-\mathrm{C} 61-\mathrm{O} 1 \mathrm{6}$ & $122.3(6)$ & $\mathrm{C} 5-\mathrm{C} 4-\mathrm{C} 2$ & $114.7(5)$ \\
\hline $\mathrm{O} 41-\mathrm{C} 4-\mathrm{C} 2$ & $107 \cdot 3(4)$ & $\mathrm{O} 41-\mathrm{C} 4-\mathrm{C} 5$ & $105.7(4)$ \\
\hline $\mathrm{C} 51-\mathrm{O} 51-\mathrm{C} 5$ & $118.9(4)$ & $\mathrm{C} 7-\mathrm{O} 71-\mathrm{C} 71$ & $116.9(4)$ \\
\hline $\mathrm{C} 7-\mathrm{C} 6-\mathrm{Cs}$ & $114.2(5)$ & $\mathrm{O} 61-\mathrm{C} 6-\mathrm{C} 5$ & 106.9 (4) \\
\hline $\mathrm{O} 61-\mathrm{C} 6-\mathrm{C} 7$ & $109 \cdot 3(4)$ & $\mathrm{C} 61-\mathrm{O} 61-\mathrm{C} 6$ & $116.8(4)$ \\
\hline $\mathrm{O} 71-\mathrm{C} 7-\mathrm{C} 6$ & $109.0(4)$ & $\mathrm{C} 6-\mathrm{C} 7-\mathrm{C} 8$ & $110.9(5)$ \\
\hline $\mathrm{O} 71-\mathrm{C} 7-\mathrm{C} 8$ & $110 \cdot 5(4)$ & $\mathrm{C} 4-\mathrm{O} 41-\mathrm{C} 41$ & $116.9(4)$ \\
\hline $\mathrm{O} 51-\mathrm{C} 51-\mathrm{C} 52$ & $112.9(6)$ & $\mathrm{O} 51-\mathrm{C} 51-\mathrm{O} 52$ & $121.7(6)$ \\
\hline $\mathrm{O} 52-\mathrm{C} 51-\mathrm{C} 52$ & $125 \cdot 4(6)$ & $\mathrm{O} 51-\mathrm{C} 5-\mathrm{C} 6$ & $105 \cdot 0(4)$ \\
\hline $\mathrm{C} 4-\mathrm{C} 5-\mathrm{C} 6$ & $112.0(5)$ & $\mathrm{C} 4-\mathrm{C} 5-\mathrm{O} 1$ & $109.0(4)$ \\
\hline $\mathrm{C} 7-\mathrm{C} 8-\mathrm{O} 81$ & $108.0(5)$ & $\mathrm{C} 8-\mathrm{O} 81-\mathrm{C} 81$ & $115.6(5)$ \\
\hline $\mathrm{C} 31-\mathrm{C} 3-\mathrm{N} 1$ & $107 \cdot 3(5)$ & $\mathrm{C} 31-\mathrm{C} 3-\mathrm{C} 2$ & $113.9(5)$ \\
\hline $\mathrm{C} 2-\mathrm{C} 3-\mathrm{N} 1$ & $105 \cdot 3(5)$ & $\mathrm{C} 4-\mathrm{C} 2-\mathrm{C} 3$ & $112.2(5)$ \\
\hline $\mathrm{C} 3-\mathrm{C} 2-\mathrm{Cl}$ & $99.6(4)$ & $\mathrm{C} 4-\mathrm{C} 2-\mathrm{C} 1$ & $114.7(5)$ \\
\hline $\mathrm{O} 41-\mathrm{C} 41-\mathrm{C} 42$ & $109 \cdot 0(6)$ & $\mathrm{C} 42-\mathrm{C} 41-\mathrm{O} 42$ & $126.4(6)$ \\
\hline $\mathrm{O} 41-\mathrm{C} 41-\mathrm{O} 42$ & $124 \cdot 6(6)$ & $\mathrm{C} 3-\mathrm{N} 1-\mathrm{N} 2$ & $113.3(5)$ \\
\hline $\mathrm{C} 2-\mathrm{C} 1-\mathrm{C} 11$ & $118.3(6)$ & $\mathrm{C} 2-\mathrm{C} 1-\mathrm{N} 11$ & $110.2(5)$ \\
\hline $\mathrm{C} 2-\mathrm{C} 1-\mathrm{N} 2$ & $104 \cdot 4(5)$ & $\mathrm{N} 11-\mathrm{Cl}-\mathrm{C} 11$ & $113.6(6)$ \\
\hline $\mathrm{N} 2-\mathrm{Cl}-\mathrm{C} 11$ & $107 \cdot 7(5)$ & $\mathrm{N} 2-\mathrm{Cl}-\mathrm{N} 1 \mathrm{I}$ & $100 \cdot 5(5)$ \\
\hline $\mathrm{N} 1-\mathrm{N} 2-\mathrm{Cl}$ & $111.6(5)$ & $\mathrm{O} 72-\mathrm{C} 71-\mathrm{C} 72$ & $125.8(6)$ \\
\hline $\mathrm{O} 71-\mathrm{C} 71-\mathrm{C} 72$ & $110.8(5)$ & $\mathrm{O} 71-\mathrm{C} 71-\mathrm{O} 72$ & $123.4(5)$ \\
\hline $\mathrm{C} 82-\mathrm{C} 81-081$ & $112.4(6)$ & $082-\mathrm{C} 81-081$ & $122.9(6)$ \\
\hline $\mathrm{O} 82-\mathrm{C} 81-\mathrm{C} 82$ & $124 \cdot 6(6)$ & $\mathrm{Cl}-\mathrm{N} 11-\mathrm{O} 12$ & $117.3(7)$ \\
\hline $\mathrm{Cl}-\mathrm{N} 11-\mathrm{O} 11$ & $116 \cdot 7(6)$ & $\mathrm{O} 11-\mathrm{N} 11-\mathrm{O} 12$ & $126.0(7)$ \\
\hline
\end{tabular}

amplitude $q=0.244$ (6) $\AA$ and a phase angle $\varphi=$ $-149.9(14)^{\circ}$ for the sequence $\mathrm{C} 1-\mathrm{C} 2-\mathrm{C} 3-\mathrm{N} 1-$ N2. The asymmetry parameter (Nardelli, 1983a) $\Delta C_{s}(\mathrm{C} 2)=0.021$ (3) indicates approximate mirror symmetry in the ring.

The five acetoxy groups are planar and their torsion angles with respect to the main chain are $\mathrm{C} 2-\mathrm{C} 4-\mathrm{O} 41-\mathrm{C} 41=108 \cdot 1$ (5), $\mathrm{C} 4-\mathrm{C} 5-\mathrm{O} 51-$ $\mathrm{C} 51=-119.0(5), \quad \mathrm{C} 5-\mathrm{C} 6-\mathrm{O} 61-\mathrm{C} 61=$ $-129 \cdot 3(5), \quad \mathrm{C} 6-\mathrm{C} 7-\mathrm{O} 71-\mathrm{C} 71=132.8(5)$ and $\mathrm{C} 7-\mathrm{C} 8-\mathrm{O} 81-\mathrm{C} 81=146.7(5)^{\circ}$. The Newman projections corresponding to $\mathrm{C}-\mathrm{C}$ bonds of the chain are shown in Fig. 2 from which the configuration of the sugar can be deduced.

The crystal cohesion is mainly due to van der Waals interactions but two intermolecular contacts may be considered as hydrogen bonds. These interactions are: $\mathrm{C} 72-\mathrm{H} 723 \cdots \mathrm{O} 42\left(x-\frac{1}{2},-y+\frac{1}{2},-z\right)$ linking molecules related by a screw axis parallel to

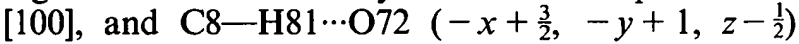
linking molecules related by a screw axis along [001]. Details of these contacts are: C72-H723 $=1.06(5)$, $\mathrm{C} 72 \cdots \mathrm{O} 42=3.448(9), \quad \mathrm{H} 723 \cdots \mathrm{O} 42=2.44(6) \AA$, $\mathrm{C} 72-\mathrm{H} 723 \cdots \mathrm{O} 42=159(4)^{\circ}$ and $\mathrm{C} 8-\mathrm{H} 81=$

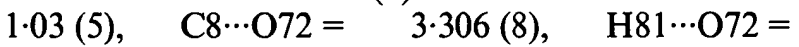
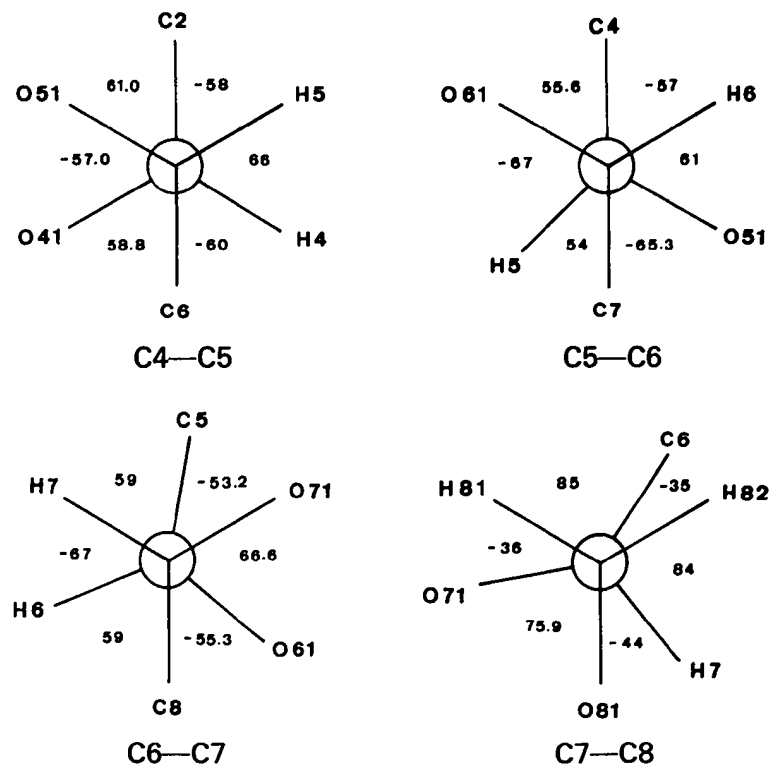

Fig. 2. Newman projections through the $\mathrm{C}-\mathrm{C}$ bonds of the sugar chain. Standard deviations in torsion angles involving non-H atoms are in the range $0.5-0.8^{\circ}$.

$2.35(6) \AA, \quad \mathrm{C} 8-\mathrm{H} 81 \cdots \mathrm{O} 72=154(4)^{\circ}$. No other contacts satisfy the criteria of Taylor \& Kennard (1982) for hydrogen bonds. The molecular geometry and crystal packing were computed by $P A R S T$ (Nardelli, 1983b).

The authors thank Professor J. A. Galbis for supplying the crystals and for discussions on chemical aspects, and Professor A. Lopez-Castro for collecting the diffractometer data. This work is part of a research project supported by the CAICYT of the Spanish Government and by the CSIC.

\section{References}

Cremer, D. \& Pople, J. A. (1975). J. Am. Chem. Soc. 97, 1354-1358.

International Tables for X-ray Crystallography (1974). Vol. IV. Birmingham: Kynoch Press. (Present distributor Kluwer Academic Publishers, Dordrecht.)

JoHNSON, C. K. (1965). ORTEP. Report ORNL-3794. Oak Ridge National Laboratory, Tennessee, USA.

Main, P., Fiske, S. J., Hull, S. E., Lessinger, L., Germain, G., DeCLerCQ, J.-P. \& WOOLFSON, M. M. (1980). MULTAN80. A System of Computer Programs for the Automatic Solution of Crystal Structures from X-ray Diffraction Data. Univs. of York, England, and Louvain, Belgium.

ManCERA, M., RodrigueZ, E., Roffe, I. \& Galbis, J. A. (1988). J. Org. Chem. 53, 5648-5651.

NARDELLI, M. (1983a). Acta Cryst. C39, 1141-1142.

Nardelli, M. (1983b). Comput. Chem. 7, 95-98.

Stewart, J. M., Machin, P. A., Dickinson, C. W., Ammon, H. L., HeCK, H. \& FlaCK, H. (1976). The XRAY76 system. Tech. Rep. TR-446. Computer Science Center, Univ. of Maryland, College Park, Maryland, USA.

TAYLOR, R. \& KenNARD, O. (1982). J. Am. Chem. Soc. 104, 5063-5070. 